\title{
Funcionalidad en el adulto mayor previa a su hospitalización a nivel nacional.
}

\author{
Nationwide Functional independence in the old adult prior to hospitalization. \\ VARELA PINEDO Luis ${ }^{1}$, CHAVEZ JIMENO Helver ${ }^{2}$, GALVEZ CANO Miguel ${ }^{3}$, MENDEZ SILVA \\ Francisco ${ }^{4}$.
}

\section{SUMMARY}

Objective: To determine the characteristics of the functionality in the Peruvian elderly adult 2 weeks before their hospitalization, also the association of the functionality with geriatrics syndromes and problems. Material and methods: On base of the information of the study "Multidisciplinary Geriatric Assessment in Peruvian Hospitals", that included 400 patients elder than 60 years; the functionality characteristics were evaluated and the functionality results were compared with the principal geriatric syndromes and problems. Results: The frequency of functional autonomy was of $53 \%$; bathing and dressing were the most compromised basic activities of the daily living in a $44.5 \%$ and $39 \%$ of the patients. There was a significant difference between functionality and age, economical situation, falls, urinary incontinence, dizziness, cognitive impairment and depression. Conclusions: The frequency of functional dependency in the pre-hospitalized elderly adult population is high; also more age, social problems, falls, urinary incontinence, dizziness, cognitive impairment and depression are associated with functional deterioration. (Rev Med Hered 2005;16:165-171).

KEY WORDS: Elderly Adult, functionality, Peru, geriatric syndromes.

\section{RESUMEN}

Objetivo: Determinar las características de la funcionalidad del adulto mayor en las 2 semanas previas a su hospitalización en el Perú, así como su asociación con otros síndromes y problemas geriátricos. Materiales $\boldsymbol{y}$ métodos: En base a la información del estudio "Valoración Geriátrica Integral en adultos mayores hospitalizados a nivel nacional", que incluyó 400 pacientes de 60 años o más; se evaluó las características de la funcionalidad y se comparó los resultados de la funcionalidad con los principales síndromes y problemas geriátricos. Resultados: Se encontró una frecuencia de autonomía funcional de 53\%, el bañarse y el vestirse fueron las Actividades Básicas de la Vida Diaria más comprometidas con $44,5 \%$ y $39 \%$ respectivamente. Se encontró relación significativa entre funcionalidad y edad, situación socioeconómica, caídas, incontinencia urinaria, mareos, desnutrición, déficit cognitivo y depresión Conclusiones: La frecuencia de dependencia funcional pre-hospitalización en la población adulta mayor es elevada. Se encontró que a mayor edad, presencia de problemas sociales, caídas, incontinencia urinaria, mareos, desnutrición, déficit cognitivo y depresión se asocia a deterioro funcional.(Rev Med Hered 2005; 16:165171).

PALABRAS CLAVE: Adulto mayor, funcionalidad, Perú, síndromes geriátricos.

Médico Asistente, Departamento de Medicina; Hospital Nacional Cayetano Heredia.

Director del Instituto de Gerontología, Universidad Peruana Cayetano Heredia.

2 Jefe del Departamento de Medicina - Servicio de Geriatría; Hospital Nacional Cayetano Heredia.

Instituto de Gerontología, Universidad Peruana Cayetano Heredia.

Médico Asistente del Hospital Nacional Cayetano Heredia. Universidad Peruana Cayetano Heredia.

Médico-Cirujano. Miembro Correspondiente del Instituto de Gerontología, Universidad Peruana Cayetano Heredia. 


\section{INTRODUCCIÓN}

El envejecimiento humano es un fenómeno universal e inevitable. Estudios demográficos revelan un aumento significativo de la población mayor de 60 años, no sólo en países desarrollados donde alcanza el 12 a $15 \%$ de la poblacion general, sino también en países en vías de desarrollo. Trabajos de proyección de crecimiento poblacional realizados en el Perú muestran que representan el $7,4 \%$ de la población y un crecimiento de dicho grupo a $12 \%$ para el año $2025(1,2)$.

La evaluación funcional como parte de la valoración integral del paciente geriátrico, para muchos, es la parte principal y la más importante. Se entiende por funcionalidad como la capacidad del sujeto de realizar las actividades de la vida diaria. La presencia del compromiso funcional practicamente no existe en los jóvenes; en cambio el compromiso de la funcionalidad en la persona mayor de 65 años puede estar presente en el $5 \%$ y en mayores de 80 años hasta en $50 \%$ o más (4). La alteración funcional puede ser un "marcador" del efecto de una enfermedad sistémica en el paciente permitiendo de esta manera su reconocimiento, establecer un diagnóstico, pronóstico, una intervención y tipo de cuidado. También resulta útil como indicador de severidad de enfermedad debido a que mide la capacidad de independencia, la cual usualmente se pierde fácilmente con las enfermedades o problemas comunes de este grupo etáreo. Dado que la población adulta mayor se encuentra en rápido crecimiento es importante conocer el impacto de los problemas de salud en su funcionalidad para mejorar su calidad de vida y reducir los costos de cuidado y atención; por ello es importante encontrar los factores que pueden relacionarse a la limitación funcional, ya que con esta información se podrán realizar políticas para identificar $\mathrm{y}$ tratar estas condiciones (3-5).

Para realizar la evaluación funcional, es necesario la utilización de escalas; estas son las de Actividades de la Vida Diaria (AVD), definida como un conjunto de acciones que una persona ejecuta todos los días o con frecuencia cotidiana, para vivir en forma autónoma e integrada en un medio ambiente y cumplir con su papel social. Se agrupan en tres categorias: actividades básicas (ABVD), instrumentales (AIVD) y avanzadas (AAVD) de la vida diaria $(3,4,6)$.

En nuestro medio se han realizado varios trabajos sobre funcionalidad. En el año 2003, el Instituto de Gerontología de la Universidad Peruana Cayetano Heredia realizó el estudio "Valoración geriátrica integral en adultos mayores hospitalizados a nivel nacional", este trabajo incluía como parte la valoración geriátrica, la evaluación funcional en las actividades básicas de la vida diaria (ABVD), es decir, el conjunto de actividades primarias que permiten a la persona movilidad, autocuidado y autonomía; usándose para este trabajo la escala de Katz (7-13).

El objetivo del presente trabajo es determinar las características de la funcionalidad del adulto mayor 2 semanas previas a su hospitalización, así como su asociación con síndromes y problemas geriátricos.

\section{MATERIALES Y MÉTODOS}

La información para el siguiente trabajo procedió del estudio "Valoración Geriátrica Integral en adultos mayores hospitalizados a nivel nacional" (13) que fue un estudio de tipo corte transversal y descriptivo, que se realizó en una población de pacientes adultos mayores de 60 años a más, hospitalizados en los servicios de medicina de diversos hospitales nivel III y IV dependientes del Ministerio de Salud y de la Seguridad Social (EsSalud) ubicados en las tres regiones naturales del país. La ubicación geográfica por muestreo de los diferentes hospitales fue la siguiente: en la costa se trabajó en los hospitales de las ciudades de Chiclayo, Chimbote, Huacho, Ica, Lima, Trujillo y Tumbes; en la sierra se trabajó en los hospitales de Arequipa, Cusco, Huamanga, Huancayo, Jauja y Puno y por último en la selva, en la ciudad de Iquitos.

Se usó el método de muestreo por clusters para hallar la muestra representativa de la población objetivo. El número estimado de camas en los servicios de medicina ocupadas por pacientes adultos mayores de los hospitales fue de 953, asimismo se determinó las unidades geográficas y sus poblaciones, eligiéndose como patrón la mas pequeña de las unidades. Se trabajó con una prevalencia estimada de presentación de los síndromes y problemas geriátricos del $50 \%$, un coeficiente de error del $4 \%$, y un intervalo de confianza de $95 \%$. El total de hospitales que ingresaron al estudio fue de 14 y el tamaño calculado de la muestra fue de 377 pacientes; sin embargo, se trabajó con 400 pacientes, con la finalidad de aumentar la confiabilidad del estudio.

El estudio original constó de 400 pacientes; se registró la funcionalidad dos semanas previas a su hospitalización en todos ellos. Se usó el índice de las actividades básicas de la vida diaria de Katz, que evalúa 6 actividades básicas de la vida diaria, las cuales son: bañarse, vestirse, usar el inodoro, levantarse, continencia y alimentación (14).

El instrumento usado para realizar la Valoración Geriátrica Integral fue el Addendum VGI validada por 
Varela L, et al (10). Este instrumento utiliza las principales escalas usadas internacionalmente, tanto para valorar los síndromes y problemas geriátricos como para la evaluación cognitiva, afectiva, y social.

Se consideró el resultado de Katz A como autonomía funcional; los resultados entre Katz B y Katz F como dependencia funcional parcial y el resultado de Katz $\mathrm{G}$ como dependencia funcional total. Para este trabajo se comparó los resultados de la funcionalidad con las variables socio-demográficas y los principales síndromes y problemas geriátricos que fueron: edad, sexo, estado civil, grado de instrucción, institución de procedencia, persona responsable, deprivación sensorial visual y auditiva, caídas, incontinencia urinaria, mareos, evaluación nutricional, evaluación social, evaluación cognitiva y evaluación afectiva.

La realización de la evaluación nutricional fue de acuerdo al índice de masa corporal (IMC) con valores ajustados para adultos mayores considerándose desnutrición un valor menor de 24 , eutrófico a un valor entre 24 y 26,9, sobrepeso entre 27 y 29,9, y obesidad a un IMC mayor de 30 (10). Para la valoración cognitiva se usó el cuestionario de salud mental de Pfeiffer; la valoración afectiva se realizó mediante la escala geriátrica de depresión de Yesavage reducida. La valoración social fue hecha con la escala de valoración socio familiar modificada (15-17).

El análisis estadístico se realizó en el programa estadístico EPI - INFO 2002 (2 ${ }^{\mathrm{a}}$ revisión). Se uso las pruebas de Chi-cuadrado para la comparación entre variables categóricas, el T-test para comparar una variable continua con una dicotómica y análisis de varianza para la comparación de variables continúas con variables con más de dos categorías. Se consideró como diferencia estadísticamente significativa una $p$ $<0,05$.

\section{RESULTADOS}

Las características socio-demográficas de la población estudiada se encuentran en el Tabla $\mathrm{N}^{\circ} 1$. La distribución por grupos etáreos fue como sigue: de 60 a 69 años, 125 pacientes $(31,25 \%)$; de 70 a 79,159 pacientes (39\%) y de 80 años a más, $119(29,75 \%)$. El 56,3\% de los pacientes tenían como cuidador a sus hijos, no se halló relación significativa entre el nivel de funcionalidad y tipo de cuidador.

Los resultados de la valoración funcional se muestran en la figura $\mathrm{N}^{\circ} 1$. Se encontró que el 46,5\% tenían algún grado de dependencia para desarrollar las actividades básicas de la vida diaria (ABVD) dos semanas antes
Tabla N¹. Características socio-demográficas de los pacientes adultos mayores hospitalizados a nivel nacional.

\begin{tabular}{|c|c|c|}
\hline & Frecuencia & Porcentaje \\
\hline \multicolumn{3}{|l|}{ Edad } \\
\hline Media + / - DS & 74,68 & + / - 8,84 años \\
\hline \multicolumn{3}{|l|}{ Sexo } \\
\hline Femenino & 202 & $50,50 \%$ \\
\hline Masculino & 198 & $49,50 \%$ \\
\hline \multicolumn{3}{|l|}{ Estado Civil } \\
\hline Casado & 202 & $50,50 \%$ \\
\hline Viudo & 152 & $38,00 \%$ \\
\hline Soltero & 35 & $8,75 \%$ \\
\hline Divorciado & 9 & $2,25 \%$ \\
\hline Desconocido & 2 & $0,50 \%$ \\
\hline \multicolumn{3}{|l|}{ Grado de Instrucción } \\
\hline Analfabeto & 142 & $35,50 \%$ \\
\hline Primaria & 170 & $42,50 \%$ \\
\hline Secundaria & 53 & $13,25 \%$ \\
\hline Superior & 35 & $8,75 \%$ \\
\hline \multicolumn{3}{|l|}{ Sistemas de salud } \\
\hline MINSA & 245 & $61,25 \%$ \\
\hline ESSALUD & 155 & $38,75 \%$ \\
\hline \multicolumn{3}{|l|}{ Familiar cuidador } \\
\hline hijo & 225 & $56,25 \%$ \\
\hline cónyuge & 54 & $13,50 \%$ \\
\hline hermano & 17 & $4,25 \%$ \\
\hline otro & 79 & $19,75 \%$ \\
\hline desconocido & 25 & $6,25 \%$ \\
\hline
\end{tabular}

Figura No1. Funcionalidad en el adulto mayor pre-hospitalización a nivel nacional, según índice de KATZ.

dependencia total

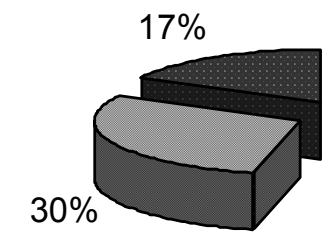

dependencia parcial

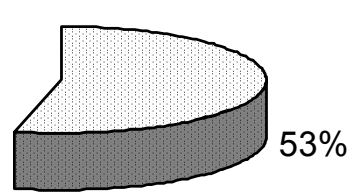

autonomia

$n=400$

a su ingreso hospitalario. El bañarse y el vestirse fueron las ABVD más comprometidas con $44,5 \%$ y $39 \%$ respectivamente (Tabla №2).

Se encontró asociación entre el grado de dependencia y edad $(\mathrm{p}<0,001)$. Se halló que el género, estado civil y el grado de instrucción no estuvieron asociados a la dependencia. Los pacientes de los hospitales MINSA tuvieron un mejor nivel de funcionalidad que los de EsSalud, pero también tuvieron un promedio menor de edad. La situación social presentó asociación con el 
Tabla No2. Funcionalidad en el adulto mayor prehospitalización a nivel nacional. Puntajes parciales segun Katz.

\begin{tabular}{cc}
\hline $\begin{array}{c}\text { Actividades básicas } \\
\text { de la vida diaria } \\
\text { (ABVD) }\end{array}$ & $\begin{array}{c}\text { Pacientes con } \\
\text { limitación en ABVD } \\
(\%)\end{array}$ \\
\hline Bañarse & $44,50 \%$ \\
Vestirse & $39,00 \%$ \\
Usar inodoro & $36,50 \%$ \\
Levantarse & $31,25 \%$ \\
Continencia & $24,50 \%$ \\
Alimentación & $21,50 \%$ \\
\hline
\end{tabular}

nivel de funcionalidad. Se encontró una relación significativa positiva entre la dependencia funcional y las caídas, la presencia de mareos, incontinencia urinaria, desnutrición, depresión (depresión leve y severa) (Tabla $\mathrm{N}^{\circ} 3$ ) y nivel cognitivo (Figura $\mathrm{N}^{\circ} 2$ ); mientras que no hubo relación entre deprivación sensorial visual o auditiva y el grado de dependencia, aunque si se encontró relación entre dependencia total $\mathrm{y}$ deprivación visual.

\section{DISCUSIÓN}

La evaluación de la capacidad funcional es el centro de la valoración del adulto mayor, siendo a veces su compromiso la única manifestación de una enfermedad subyacente, además es uno de los principales determinantes de su calidad de vida. Desde el punto de vista económico, los costos hospitalarios son más altos en pacientes dependientes. En nuestro estudio se encontró una prevalencia de $47 \%$ de dependencia ( $17 \%$ de dependencia total), valor elevado comparado con las series norteamericanas; pero muy cercanos a los
Figura $N^{\circ}$ 2. Relación significativa positiva a nivel cognitivo.

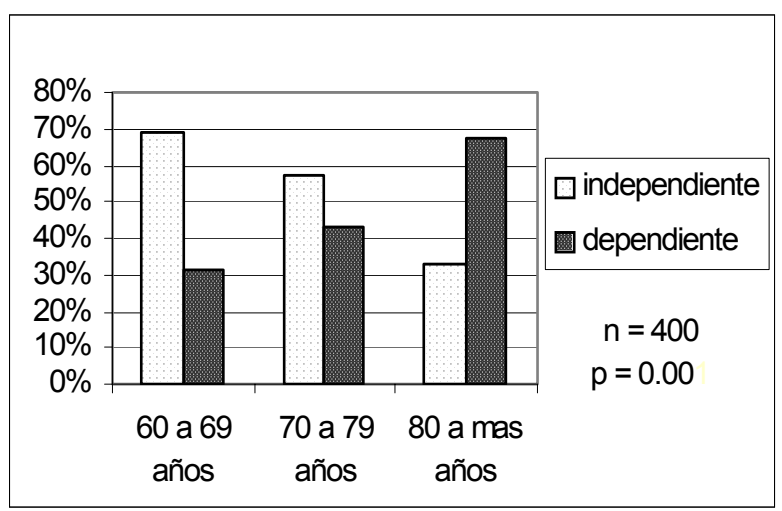

valores encontrados en estudios nacionales; esto se debe probablemente a que se evalúa la funcionalidad en un tiempo cercano a la hospitalización, con lo cual esta ya puede estar afectada por la enfermedad subyacente (11, 12,18).

Las actividades de la vida diaria más complejas presentan mayor frecuencia de compromiso; similar a lo expuesto por Katz (14) al formular el diseño de su instrumento; en donde las actividades más complejas se comprometen de manera temprana y cuando se recuperan se realizan inicialmente a través de las más simples.

Se encontró también que la prevalencia de este problema aumenta con la edad, resultado que coincide con estudios previos donde se observa que la edad cronológica es uno de los factores más importantes de deterioro funcional, con un incremento en el riesgo relativo de 2,0 por cada 10 años (18).

En nuestro trabajo no se encontró una relación

Tabla No3. Relación entre funcionalidad y síndromes geriátricos en el adulto mayor pre-hospitalización a nivel nacional.

\begin{tabular}{cccccc}
\hline & $\mathrm{n}$ & independiente & dependiente & $\mathrm{p}$ & $\mathrm{IC}$ \\
\hline Deprivación auditiva & 163 & $47,85 \%$ & $52,15 \%$ & 0,038 & $0,997-2,201$ \\
Deprivación visual & 281 & $54,45 \%$ & $45,55 \%$ & 0,317 & $0,573-1,351$ \\
$\quad$ Caídas & 159 & $40,88 \%$ & $59,12 \%$ & 0,001 & $1,555-3,522$ \\
Incontinencia urinaria & 212 & $44,34 \%$ & $55,66 \%$ & 0,001 & $1,481-3,312$ \\
$\quad$ Mareos & 160 & $47,50 \%$ & $52,50 \%$ & 0,034 & $1,072-2,225$ \\
$\quad \begin{array}{c}\text { Desnutrición } \\
\text { Riesgo/ Depresión }\end{array}$ & 218 & $48,17 \%$ & $51,83 \%$ & 0,012 & $1,179-2,593$ \\
$\quad \begin{array}{c}\text { establecida } \\
\text { Deterioro cognitivo }\end{array}$ & 144 & $54,86 \%$ & $45,14 \%$ & 0,001 & $1,433-4,877$ \\
moderado / severo & 70 & $25,71 \%$ & $74,29 \%$ & 0,001 & $3,481-11,574$ \\
\hline
\end{tabular}


significativa entre la funcionalidad y el sexo o estado civil. Este resultado es similar al de otros estudios (1921). Sin embargo, llama la atención que no se encontrará una relación significativa con el grado de instrucción, probablemente porque en nuestra muestra, así como en nuestra población, hay una elevada frecuencia de personas con bajo grado de instrucción $(18,22)$.

Se encontró que la mayor parte de los pacientes tenían por principales cuidadores a sus familiares directos y de estos la mayoría eran sus hijos. No hubo relación entre el tipo de cuidador y el nivel de funcionalidad; probablemente debido a que ser "familiar directo" si bien puede implicar tener una actitud positiva hacia el adulto mayor en las áreas afectiva y social en general, no implica que puede existir cierta indiferencia hacia el cuidado directo de la salud (23) al no tener la capacidad o conocimientos acerca del cuidado y prevención de sus enfermedades, o considerar que la pérdida de funcionalidad es parte natural del proceso de envejecimiento. Los pacientes de EsSalud presentaron un mayor grado de dependencia funcional posiblemente debido a que también presentaban mayor edad que los de MINSA.

La funcionalidad estuvo asociada a la situación social, probablemente se deba a que una mejor situación socioeconómica posibilita la prevención, el cuidado y un mejor tratamiento de las condiciones de fondo que ocasionan el deterioro funcional, a la vez la familia cuenta con mayores recursos para la rehabilitación. Según series extranjeras esta variable presenta una asociación fuerte en estudios longitudinales y trasversales $(18,24)$.

Llama la atención que el compromiso visual no este asociado a la independencia, cuando en otros estudios $(25,26)$ se considera que estas dos variables presentan una asociación fuerte; esto probablemente se deba a los métodos utilizados para evaluar esta condición en el presente estudio (auto reporte), lo cual podría haber incrementado el número de casos, con grados leves de déficit visual. Sin embargo, se encontró que en el grupo de pacientes con deterioro funcional severo existían más casos de deprivación visual. En estudios extranjeros (27) se ha observado que el compromiso auditivo se encuentra débilmente asociado a la disminución de la capacidad funcional; en nuestro trabajo no se encontró una asociación significativa.

Las caídas están fuertemente asociadas al compromiso funcional, esto se puede explicar a que, su presencia puede ocasionar deterioro funcional por lesión, que el paciente limite su actividad tratando de evitar nuevas caídas, como un síndrome post-caída o que el deterioro funcional pre-existente haya ocasionado una caída.

Existe relación significativa con la presencia de incontinencia urinaria, la cual puede deberse a que esta condición puede tener una repercusión psicológica que limite su actividad social y afectiva, las cuales a su vez disminuyan los niveles funcionalidad; este síndrome también puede implicar un estado de salud disminuido, asociado a un déficit funcional previo.

Las razones de asociación entre mareos y funcionalidad son similares a las propuestas para el caso de las caídas, aunque para la presencia de mareos esta relación es menos fuerte; esto puede deberse a que en el caso de mareos aun no se ha producido el evento y solo genera que el paciente limite su actividad tratando de evitar las caídas.

La relación significativa encontrada con desnutrición puede ser explicada debido a que esta condición generalmente implica la disminución de fuerza muscular, alteraciones del equilibrio y pobre estado de salud que afecta la funcionalidad. En series extranjeras se observan resultados similares al respecto de un pobre estado nutricional, aunque también se encuentra una relación entre deterioro funcional y sobrepeso, que no se da en nuestro estudio probablemente debido a la poca cantidad de pacientes obesos y con sobrepeso hallados (28).

Se encontró una asociación significativa con deterioro cognitivo, esta alteración puede provocar un falta de interés por realizar actividades, puede haber también pérdida de capacidad para realizar ciertas funciones y que los familiares al querer proteger al adulto mayor con este problema, limiten sus actividades (Figura $\mathrm{N}^{\circ} 3$ ). En los estudios extranjeros se encuentra que esta

\section{Figura No3. Funcionalidad en el adulto mayor pre-hospitalización a nivel nacional, según nivel cognitivo.}

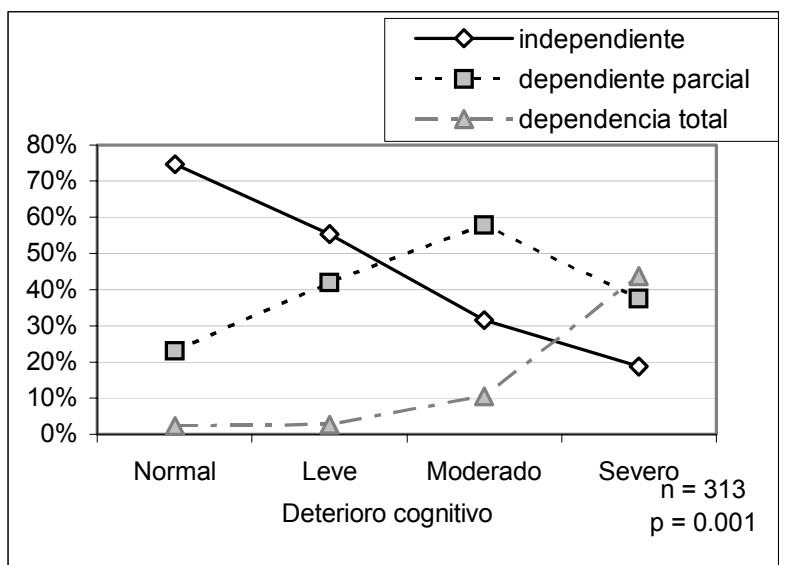


asociación es fuerte, siendo la pérdida de orientación y memoria los items de mayor valor predictivo (29).

La relación encontrada entre funcionalidad y depresión puede deberse a que esta última al causar desinterés y anhedonia disminuya las actividades que el paciente realiza. También es posible que la limitación funcional previa cause el estado depresivo. En otros estudios se encuentra también esta asociación $(30,31)$.

Si bien el presente trabajo es realizado solamente a nivel hospitalario y sus resultados no pueden aplicarse a la población adulta mayor en general; se encontró una alta frecuencia de dependencia funcional dos semanas antes a la hospitalización, hallándose asociación entre el nivel de funcionalidad y edad, situación social, presencia de deterioro cognitivo, depresión, caídas, desnutrición, mareos, e incontinencia urinaria. Se requieren nuevos estudios con seguimiento y comunitarios, para esclarecer los factores precipitantes y etiológicos en el deterioro de la funcionalidad así como para conocer su impacto en la calidad de vida de los adultos mayores de nuestra población. Esta es una tarea importante para desarrollar programas de promoción y prevención de la salud en busca de alcanzar un envejecimiento saludable y activo $(32,33)$.

\section{Correspondencia:}

Luis Varela Pinedo

Instituto de Gerontología

Universidad Peruana Cayetano Heredia

Av. Honorio Delgado 430

San Martin de Porres

Lima, Perú.

Correo electrónico: igero@upch.edu.pe

\section{REFERENCIAS BIBLIOGRÁFICAS}

1. Naciones Unidas. Documento Introductorio de la Segunda Asamblea Mundial sobre el Envejecimiento: Consideraciones Demográficas. Viena: Naciones Unidas - Asamblea sobre envejecimiento; 1982.

2. Instituto Nacional de Estadística e Informática (INEI). Perfil sociodemográfico de la tercera edad. Lima Perú: Instituto Nacional de Estadística e Informática;1995.

3. Varela L. Principios de Geriatría y Gerontología. 1ra Edición. Lima: Centro Editorial de la Universidad Peruana Cayetano Heredia;2003:1-5.

4. Hazzard W, Reubin A. Principles of geriatric medicine and gerontoly. McGraw Hill; 1999: 1535-1546.

5. Dunlop D, Manheim L, Sohn M. Incidence of functional limitation in older adults: The impact of gender, race, and chronic conditions. Arch Phys Med Rehab 2002; 83: 964-971.
6. Tallis RC, Fillit HM. Brocklehurst's textbook of geriatric medicine and gerontoly. $6^{\text {th }}$ edition. Churchill Livingston; 2003:291-299.

7. Chu M. Salud y autonomía en relación al medio circundante de la población de 65 años y más en Lima y Cuzco. Tesis para grado de Magister. Lima, Peru. Universidad Peruana Cayetano Heredia, 1990.

8. Sandoval L, Varela L. Estudio comparativo de funcionalidad en pacientes adultos mayores ambulatorios y hospitalizados. Rev Med Hered 1988; 9(4):138-42.

9. Hardy G. Estudio comparativo de funcionalidad en una serie de pacientes adultos mayores ambulatorios y hospitalizados. Tesis para grado de Especialista en Medicina Física y Rehabilitación. Lima, Perú. Universidad Nacional Mayor de San Marcos, 1999.

10. Varela L, Silicani A, Chavez H, Chigne O. Valoración geriátrica integral: propuesta de addendum a la historia clínica. Diagnóstico 2000; 39(3):135-47.

11. Villar D, Varela L, Chigne O. Evaluación geriátrica integral en pacientes hospitalizados y ambulatorios. Bol Soc Perú Med Interna 2000; 13(3): 143-50.

12. Alva J. Evaluacion geriatrica integral en pacientes hospitalizados y ambulatorios del Hospital Nacional Edgardo Rebagliati Martins: Morbilidad y problemas asociados. Tesis para grado de Especialista en Medicina Interna. Lima, Perú. Universidad Nacional Mayor de San Marcos, 2001.

13. Varela L, Chavez H, Herrera A, Ortiz P, Chigne O. Valoración Geriátrica Integral en adultos mayores hospitalizados a nivel nacional. Diagnóstico 2004;.43 (2):57-63.

14. Katz S, Ford A, Moskowitz R, Studies of illness in the Aged. JAMA 1963; 185 (12):94-99.

15. Pfeiffer E. A short portable mental status questionnaire for the assessment of organic brain deficit in elderly patients. J Am Geriatr Soc 1975; 23 (2): 39-41.

16. Sheikh JL, Yesavage JA. Geriatric Depression Scale (GDS): Recent evidence and development of a shorter version. In: Brink TL. Clinical gerontology: A guide to assessment and intervention. New York: Haworth Press; 1986:165.

17. Díaz M, Domínguez O, Toyos G. Resultados de la aplicación de una escala de valoración socio-familiar en atención primaria. Revista Española de Geriatría y Gerontología 1994; 29(4):239-45.

18. Guralnik JM, LaCroix AZ, Abbot RD. Maintaining mobility in late life I. Demographic characteristics and chronic conditions. Am J Epidemiol 1993; 137: 845857.

19. Strawbridge WJ, Kaplan GA, Camacho T, Cohen RD. The dynamics of disability and functional change in an elderly cohort: results from the Alameda County study. J Am Geriatr Soc 1992; 40: 799-806.

20. Arber S, Ginn J. Gender and inequalities in health in later life. Soc Sci Med 1993;36: 33-46.

21. Goldman N, Korenman S, Weinstein R. Marital status and health among the elderly. Soc Sci Med 1995; 40: $1717-1730$

22. Snowdon DA, Ostwald SK, Kane RL. Education 
survival and independence in elderly catholic sisters: 1936-1988. Am J Epidemiol 1998; 130: 999-1012.

23. Perez F. Estudio de las actitudes de la familia hacia el anciano en dos comunidades de Condevilla - San Martín de Porres. Tesis de bachiller en Enfermería. Lima, Perú. Universidad Peruana Cayetano Heredia, 1986.

24. Boult Ch, Kane RL, Louis TA, Boult L, McCaffrey D. Chronic conditions that lead to functional limitation in the elderly. J Gerontol Med Sci 1994; 49: 28-36.

25. Salive ME, Guralnik J, Glynn RJ, Christen W, Wallace RB, Ostfeld AM. Association of visual impairment with mobility and physical function. J Am Geriatr Soc 1994; 42: 287-292.

26. Rubin GS, Bandeen Roche K, Prasada-Rao P, Fried LP. Visual impairment and disability in older adults. Optom Vis Sci 1994; 71: 750-760.

27. Furner SE, Rudberg MA, Cassel CK. Medical conditions differentially affect the development of IADL disability: implications for medical care and research. Gerontologist 1995; 35(4): 444-450.
28. Launer LJ, Harris T, Rumpel C, Madans J. Body mass index, weight change and risk of mobility disability in middle-aged and older women. JAMA 1994; 271(14), 1093-1098.

29. Gill TM, Williams CS, Richardson ED, Berkman LF, Tinneti ME. A predictive model for ADL dependence community -living older adults based on a reduced se of cognitive status items. J Am Geriatr Soc 1997; 45: 441-445.

30. Gallo JJ, Rabins PV, Lyketsos CG, Tien AY, Anthony JC. Depression without sadness: functional outcomes of nondysphoric depression in later life. J Am Geriatr Soc 1997; 43: 603-609.

31. Penninx, BW, Guralnik JM, Ferruci L, Simonsick EM, Deeg, DJ, Wallace RB. Depressive symptoms and physical decline in community-dwelling older persons. JAMA 1998; 279: 1720-1726.

32. Varela L. Geriatría: Valoración geriátrica integral. Diagnóstico 2003; 42 (2): 73-80.

33. Cobbs EL, Duthie EH. Geriatrics Review Syllabus: a core curriculum in geriatric medicine. Fifth Edition. Malden, MA: Blackwell Publish; 2003.

Recibido: 01/02/05

Aceptado para publicación: 21/05/05 